\title{
The solutions of one type $q$-difference functional system
}

\author{
Yonglin $\mathrm{Xu}^{1 *}$, Dengli Han ${ }^{2}$, Xiaohong Fan ${ }^{3}$, Gang Wang ${ }^{3}$ and Hua Zhong ${ }^{3}$
}

\author{
"Correspondence: \\ xuyonglin000@163.com \\ 'Mathematics and Computer \\ College, Northwest Minorities \\ University, Lanzhou, Gansu 730030, \\ China \\ Full list of author information is \\ available at the end of the article
}

\begin{abstract}
In this paper, we study the functional system on q-difference equations, our results can give estimates on the proximity functions and the counting functions of the solutions of $q$-difference equations system. This implies that solutions have a relatively large number of poles. The main results in this paper concern $q$-difference equations to the system of $q$-difference equations.
\end{abstract}

MSC: Primary 30D35; secondary 39B32; 39A13; 39B12

Keywords: functional system; $q$-difference equations; zero order; difference Nevanlinna theory

\section{Introduction and main results}

A function $f(z)$ is called meromorphic if it is analytic in the complex plane $\mathbb{C}$ except at isolate poles. In what follows, we assume that the reader is familiar with the basic notion of Nevanlinna's value distribution theory, see [1] and [2].

Let us consider the $q$-difference polynomial case. Let $d_{j} \in \mathbb{C}$ for $j=1, \ldots, n$, and let $I_{q}$ be a finite set of multi-indexes $\gamma=\left(\gamma_{0}, \ldots, \gamma_{n}\right)$. A $q$-difference polynomial of a meromorphic function $w(z)$ is defined as follows:

$$
\begin{aligned}
P(z, w) & =P\left(z, w(q z), w\left(q^{2} z\right), \ldots, w\left(q^{n} z\right)\right) \\
& =\sum_{\gamma \in I_{q}} a_{\gamma}(z) w(z)^{\gamma_{0}} w(q z)^{\gamma_{1}} \cdots w\left(q^{n} z\right)^{\gamma_{n}},
\end{aligned}
$$

where $q \in \mathbb{C}\{0\}$, and the coefficients $a_{\gamma}(z)$ are small meromorphic functions with respect to $w(z)$ such that $T\left(r, a_{\gamma}\right)=o(T(r, w))$ on a logarithmic density 1 , denoted by $S_{q}(r, w)$. The total degree of $P(z, w)$ in $w(z)$ and the $q$-shifts of $w(z)$ is denoted by $\operatorname{deg}_{w}^{q}(P)$, and the order of zero of $P\left(z, x_{0}, x_{1}, \ldots, x_{n}\right)$, as a function of $x_{0}$ at $x_{0}=0$, is denoted as $\operatorname{ord}_{0}^{q}(P)$, which can be found, e.g., in [3]. Moreover, the weight of difference polynomial (1.1) is defined by

$$
K_{q}(P)=\max _{\gamma \in I_{q}}\left\{\sum_{j=1}^{n} \gamma_{j}\right\},
$$

where $\gamma$ and $I_{q}$ are the same as in (1.1) above. The $q$-difference polynomial $P(z, w)$ is said to be homogeneous with respect to $w(z)$ if the degree $d_{\gamma}=\gamma_{0}+\cdots+\gamma_{n}$ of each term in the sum (1.1) is non-zero and the same for all $\gamma \in I_{q}$.

We recall the following result of Zhang et al. [4, Theorem 1].

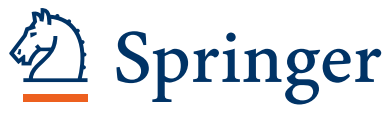

○2014 Xu et al.; licensee Springer. This is an Open Access article distributed under the terms of the Creative Commons Attribution License (http://creativecommons.org/licenses/by/2.0), which permits unrestricted use, distribution, and reproduction in any medium, provided the original work is properly cited. 
Theorem A Let $w(z)$ be a zero-order meromorphic solution of

$$
H(z, w) P(z, w)=Q(z, w)
$$

where $P(z, w)$ is a homogeneous q-difference polynomial with polynomial coefficients, and $H(z, w)$ and $Q(z, w)$ are polynomials in $w(z)$ with polynomial coefficients having no common factors. If

$$
\max \left\{\operatorname{deg}_{w}^{q}(H), \operatorname{deg}_{w}^{q}(Q)-\operatorname{deg}_{w}^{q}(P)\right\}>\min \left\{\operatorname{deg}_{w}^{q}(P), \operatorname{ord}_{0}^{q}(Q)\right\}-\operatorname{ord}_{0}^{q}(P)
$$

then $N(r, w) \neq S_{q}(r, w)$, where $\operatorname{ord}_{0}^{q}(P)$ denotes the order of zero of $P\left(z, x_{0}, x_{1}, \ldots, x_{n}\right)$, as a function of $x_{0}$ at $x_{0}=0$.

Now let us introduce some notation. Let $q_{j} \in \mathbb{C} \backslash\{0$,$\} for j=1, \ldots, n$, and let $I$ and $J$ be a finite set of multi-indexes $I=\left(i_{0}, \ldots, i_{n}\right)$ and $J=\left(j_{0}, \ldots, j_{n}\right)$. Two $q$-difference polynomials of a meromorphic function $w(z)$ are defined as follows:

$$
\begin{aligned}
\Omega_{1}\left(z, w_{1}, w_{2}\right) & =\Omega_{1}\left(z, w_{1}(z), w_{2}(z), w_{1}\left(q_{1} z\right), w_{2}\left(q_{1} z\right), \ldots, w_{1}\left(q_{n} z\right), w_{2}\left(q_{n} z\right)\right) \\
& =\sum_{i \in I} a_{i}(z) \prod_{k=1}^{2} w_{k}(z)^{k_{i_{0}}} w_{k}\left(q_{1} z\right)^{k_{i_{1}}} \cdots w_{k}\left(q_{n} z\right)^{k_{i_{n}}}
\end{aligned}
$$

and

$$
\begin{aligned}
\Omega_{2}\left(z, w_{1}, w_{2}\right) & =\Omega_{2}\left(z, w_{1}(z), w_{2}(z), w_{1}\left(q_{1} z\right), w_{2}\left(q_{1} z\right), \ldots, w_{1}\left(q_{n} z\right), w_{2}\left(q_{n} z\right)\right) \\
& =\sum_{j \in J} b_{j}(z) \prod_{k=1}^{2} w_{k}(z)^{k_{i_{0}}} w_{k}\left(q_{1} z\right)^{k_{i_{1}}} \ldots w_{k}\left(q_{n} z\right)^{k_{i_{n}}},
\end{aligned}
$$

where the coefficients $a_{i}(z)$ and $b_{j}(z)$ are small with respect to $w_{1}(z)$ and $w_{2}(z)$ in the sense that $T\left(r, a_{i}\right)=o\left(T\left(r, w_{k}\right)\right)$ and $T\left(r, b_{j}\right)=o\left(T\left(r, w_{k}\right)\right), k=1,2$, on a set of logarithmic density 1 , as $r$ tends to infinity outside of an exceptional set $E$ of finite logarithmic measure

$$
\lim _{r \rightarrow \infty} \int_{E \cap[1, r)} \frac{d t}{t}<\infty
$$

The weights of $\Omega_{1}\left(z, w_{1}, w_{2}\right)$ and $\Omega_{2}\left(z, w_{1}, w_{2}\right)$ in $w_{1}(z), w_{2}(z)$ are denoted by

$$
\lambda_{11}=\max _{i}\left\{\sum_{l=0}^{n} i_{1 l}\right\}, \quad \lambda_{12}=\max _{i}\left\{\sum_{l=0}^{n} i_{2 l}\right\}
$$

and

$$
\lambda_{21}=\max _{j}\left\{\sum_{l=0}^{n} i_{1 l}\right\}, \quad \lambda_{22}=\max _{j}\left\{\sum_{l=0}^{n} i_{2 l}\right\} .
$$

The purpose of this paper is to study the problem of the properties of Nevanlinna counting functions and proximity functions of meromorphic solutions of a type of systems of 
$q$-difference equations of the following form:

$$
\left\{\begin{array}{l}
\Omega_{1}\left(z, w_{1}, w_{2}\right)=R_{1}\left(z, w_{1}\right), \\
\Omega_{2}\left(z, w_{1}, w_{2}\right)=R_{2}\left(z, w_{2}\right),
\end{array}\right.
$$

where

$$
R_{1}\left(z, w_{1}\right)=\frac{P_{1}\left(z, w_{1}\right)}{Q_{1}\left(z, w_{1}\right)}=\frac{\sum_{i=0}^{p_{1}} a_{i}(z) w_{1}^{i}}{\sum_{j=0}^{q_{1}} b_{j}(z) w_{1}^{j}}
$$

and

$$
R_{2}\left(z, w_{2}\right)=\frac{P_{2}\left(z, w_{2}\right)}{Q_{2}\left(z, w_{2}\right)}=\frac{\sum_{i=0}^{p_{2}} c_{i}(z) w_{2}^{i}}{\sum_{j=0}^{q_{2}} d_{j}(z) w_{2}^{j}}
$$

the coefficients $\left\{a_{i}(z)\right\},\left\{b_{i}(z)\right\},\left\{c_{i}(z)\right\},\left\{d_{i}(z)\right\}$ are meromorphic functions and small functions. The order of zero of $\Omega_{j}\left(z, x_{0}, \ldots, x_{n}\right)$, as a function of $x_{0}$ at $x_{0}=0$, is denoted by $\operatorname{ord}_{0}\left(\Omega_{j}\right)$. The $q$-difference polynomial $\Omega_{k}\left(z, w_{1}, w_{2}\right), k=1,2$, is said to be homogeneous with respect to $w_{k}(z)$ if the degree $d_{k}=i_{k 0}+\cdots+i_{k n}$ of each term in the sum is non-zero and the same for all $i \in I$. Finally, the order of growth of a meromorphic solution $\left(w_{1}, w_{2}\right)$ is defined by

$$
\rho\left(w_{1}, w_{2}\right)=\max \left\{\rho\left(w_{1}\right), \rho_{2}\left(w_{2}\right)\right\},
$$

where

$$
\rho\left(w_{k}\right)=\limsup _{r \rightarrow \infty} \frac{\log T\left(r, w_{k}\right)}{\log r}, \quad k=1,2 .
$$

In this paper, the main results are as follows.

Theorem 1 Let $\left(w_{1}, w_{2}\right)$ be a zero-order meromorphic solution of system (1.2), where $\Omega_{k}\left(z, w_{1}, w_{2}\right)(k=1,2)$ are homogeneous $q$-difference polynomials in $w_{1}$ and $w_{2}$, respectively, with meromorphic coefficients, and $P_{k}\left(z, w_{k}\right)$ and $Q\left(z, w_{k}\right), k=1,2$, are polynomials in $w_{k}(z)$ with meromorphic coefficients having no common factors. If

$$
\max \left\{q_{1}, p_{1}-\lambda_{11}\right\}>\min \left\{\lambda_{11}, \operatorname{ord}_{w_{1}}\left(P_{1}\right)\right\}-\operatorname{ord}_{w_{1}}\left(\Omega_{1}\right)+\lambda_{12}
$$

and

$$
\max \left\{q_{2}, p_{2}-\lambda_{22}\right\}>\min \left\{\lambda_{22}, \operatorname{ord}_{w_{2}}\left(P_{2}\right)\right\}-\operatorname{ord}_{w_{2}}\left(\Omega_{2}\right)+\lambda_{21},
$$

then $N\left(r, w_{1}\right)=S_{q}\left(r, w_{1}\right)$ and $N\left(r, w_{2}\right)=S_{q}\left(r, w_{2}\right)$ cannot hold both at the same time, possibly outside of an exceptional set of finite logarithmic measure.

Theorem 2 Let $\left(w_{1}, w_{2}\right)$ be a zero-order meromorphic solution of system (1.2), where $\Omega_{k}\left(z, w_{1}, w_{2}\right)(k=1,2)$ are homogeneous $q$-difference polynomials in $w_{1}$ and $w_{2}$, respectively, 
with meromorphic coefficients, and $P_{k}\left(z, w_{k}\right)$ and $Q\left(z, w_{k}\right), k=1,2$, are polynomials in $w_{k}(z)$ with meromorphic coefficients having no common factors,

$$
A=2 \lambda_{11}-\left(\max \left\{p_{1}, q_{1}+\lambda_{11}\right\}-\min \left\{\lambda_{11}, \operatorname{ord}_{w_{1}}\left(\Omega_{1}\right)\right\}\right)
$$

and

$$
B=2 \lambda_{22}-\left(\max \left\{p_{2}, q_{2}+\lambda_{22}\right\}-\min \left\{\lambda_{22}, \operatorname{ord}_{w_{2}}\left(\Omega_{2}\right)\right\}\right) .
$$

If $A<0, B<0$ and $A B>9 \lambda_{21} \lambda_{12}$, then $m\left(r, w_{k}\right)=S_{q}\left(r, w_{k}\right)(k=1,2)$, where $r$ runs to infinity outside of an exceptional set of finite logarithmic measure.

\section{Some lemmas}

Lemma 1 ([5], Theorem 1.2) Let $f(z)$ be a non-constant zero-order meromorphic function, and $q \in \mathbb{C} \backslash\{0\}$. Then

$$
m\left(r, \frac{f(q z)}{f(z)}\right)=S_{q}(r, f) .
$$

Lemma 2 ([6], Lemma 4) If $T: \mathbb{R}^{+} \rightarrow \mathbb{R}^{+}$is a piecewise continuous increasing function such that

$$
\lim _{r \rightarrow \infty} \frac{\log T(r)}{\log r}=0
$$

then the set

$$
E:=\left\{r: T\left(C_{1} r\right) \geq C_{2} T(r)\right\}
$$

has logarithmic density 0 for all $C_{1}>1$ and $C_{2}>1$.

\section{Proof of Theorem 1}

Since $\Omega_{k}\left(z, w_{1}, w_{2}\right)$ are homogeneous in $w_{1}$ and $w_{2}$, respectively, it follows by Lemma 1 that

$$
m\left(r, \frac{\Omega_{1}\left(z, w_{1}, w_{2}\right)}{w_{1}^{\lambda_{11}}}\right) \leq \lambda_{12} m\left(r, w_{2}\right)+S_{q}\left(r, w_{1}\right)
$$

and

$$
m\left(r, \frac{\Omega_{2}\left(z, w_{1}, w_{2}\right)}{w_{2}^{\lambda_{22}}}\right) \leq \lambda_{21} m\left(r, w_{1}\right)+S_{q}\left(r, w_{2}\right)
$$

for all $r$ outside of an exceptional set of finite logarithmic measure. Moreover, from (1.2), we have

$$
\begin{aligned}
T\left(r, \frac{\Omega_{1}\left(z, w_{1}, w_{2}\right)}{w_{1}^{\lambda_{11}}}\right)= & T\left(r, \frac{P_{1}\left(z, w_{1}\right)}{Q_{1}\left(z, w_{1}\right) w_{1}^{\lambda_{11}}}\right) \\
= & \left(\max \left\{p_{1}, q_{1}+\lambda_{11}\right\}-\min \left\{\lambda_{11}, \operatorname{ord}_{w_{1}}\left(P_{1}\right)\right\}\right) T\left(r, w_{1}\right) \\
& +S_{q}\left(r, w_{1}\right)
\end{aligned}
$$


and

$$
\begin{aligned}
T\left(r, \frac{\Omega_{2}\left(z, w_{1}, w_{2}\right)}{w_{2}^{\lambda_{22}}}\right)= & T\left(r, \frac{P_{2}\left(z, w_{2}\right)}{Q_{2}\left(z, w_{2}\right) w_{2}^{\lambda_{22}}}\right) \\
= & \left(\max \left\{p_{2}, q_{2}+\lambda_{22}\right\}-\min \left\{\lambda_{22}, \operatorname{ord}_{w_{2}}\left(P_{2}\right)\right\}\right) T\left(r, w_{2}\right) \\
& +S_{q}\left(r, w_{2}\right)
\end{aligned}
$$

where $r$ approaches infinity outside of an exceptional set of finite logarithmic measure. By combining (3.1) and (3.3), (3.2) and (3.4), respectively, it follows that

$$
\begin{aligned}
N\left(r, \frac{\Omega_{1}\left(z, w_{1}, w_{2}\right)}{w_{1}^{\lambda_{11}}}\right) \geq & \left(1+\lambda_{12}+\lambda_{11}-\operatorname{ord}_{w_{1}}\left(\Omega_{1}\right)\right) T\left(r, w_{1}\right) \\
& -\lambda_{12} m\left(r, w_{2}\right)+S_{q}\left(r, w_{1}\right)
\end{aligned}
$$

and

$$
\begin{aligned}
N\left(r, \frac{\Omega_{2}\left(z, w_{1}, w_{2}\right)}{w_{2}^{\lambda_{22}}}\right) \geq & \left(1+\lambda_{21}+\lambda_{22}-\operatorname{ord}_{w_{2}}\left(\Omega_{2}\right)\right) T\left(r, w_{1}\right) \\
& -\lambda_{21} m\left(r, w_{1}\right)+S_{q}\left(r, w_{2}\right) .
\end{aligned}
$$

From Lemma 2, we have

$$
\begin{aligned}
& N\left(r, \frac{\Omega_{1}\left(z, w_{1}, w_{2}\right)}{w_{1}^{\operatorname{ord}_{w_{1}}\left(\Omega_{1}\left(z, w_{1}, w_{2}\right)\right)}}\right) \\
& \quad \leq\left(\lambda_{11}-\operatorname{ord}_{w_{1}}\left(\Omega_{1}\right)\right) N\left(q r, w_{1}\right)+\lambda_{12} N\left(q r, w_{2}\right)+S_{q}\left(r, w_{1}\right) \\
& \quad=\left(\lambda_{11}-\operatorname{ord}_{w_{1}}\left(\Omega_{1}\right)\right) N\left(r, w_{1}\right)+\lambda_{12} N\left(r, w_{2}\right)+S_{q}\left(r, w_{1}\right)+S_{q}\left(r, w_{2}\right)
\end{aligned}
$$

and

$$
\begin{aligned}
& N\left(r, \frac{\Omega_{2}\left(z, w_{1}, w_{2}\right)}{\left.w_{1}^{\operatorname{ord}_{w_{2}}\left(\Omega_{2}\left(z, w_{1}, w_{2}\right)\right)}\right)}\right. \\
& \quad \leq\left(\lambda_{22}-\operatorname{ord}_{w_{2}}\left(\Omega_{2}\right)\right) N\left(q r, w_{2}\right)+\lambda_{21} N\left(q r, w_{1}\right)+S_{q}\left(r, w_{2}\right) \\
& \quad=\left(\lambda_{22}-\operatorname{ord}_{w_{2}}\left(\Omega_{2}\right)\right) N\left(r, w_{2}\right)+\lambda_{11} N\left(r, w_{1}\right)+S_{q}\left(r, w_{1}\right)+S_{q}\left(r, w_{2}\right) .
\end{aligned}
$$

Therefore,

$$
\begin{aligned}
& N\left(r, \frac{\Omega_{1}\left(z, w_{1}, w_{2}\right)}{w_{1}^{\lambda_{11}}}\right) \leq N\left(r, \frac{\Omega_{1}\left(z, w_{1}, w_{2}\right)}{w_{1}^{\operatorname{ord}_{w_{1}}\left(\Omega_{1}\left(z, w_{1}, w_{2}\right)\right)}}\right)+N\left(r, \frac{1}{w_{1}^{\lambda_{11}-\operatorname{ord}_{w_{1}}\left(\Omega_{1}\right)}}\right) \\
& \leq\left(\lambda_{11}-\operatorname{ord}_{w_{1}}\left(\Omega_{1}\right)\right) N\left(r, w_{1}\right)+\lambda_{12} N\left(r, w_{2}\right) \\
& +T\left(r, \frac{1}{w_{1}^{\lambda_{11-}-\operatorname{ord}_{w_{1}}\left(\Omega_{1}\right)}}\right)+S_{q}\left(r, w_{1}\right)+S_{q}\left(r, w_{2}\right) \\
& \leq\left(\lambda_{11}-\operatorname{ord}_{w_{1}}\left(\Omega_{1}\right)\right) N\left(r, w_{1}\right)+\lambda_{12} N\left(r, w_{2}\right) \\
& +\left(\lambda_{11}-\operatorname{ord}_{w_{1}}\left(\Omega_{1}\right)\right) T\left(r, w_{1}\right)+S_{q}\left(r, w_{2}\right)+S_{q}\left(r, w_{2}\right)
\end{aligned}
$$


and

$$
\begin{aligned}
N\left(r, \frac{\Omega_{2}\left(z, w_{1}, w_{2}\right)}{w_{2}^{\lambda_{22}}}\right) \leq & N\left(r, \frac{\Omega_{2}\left(z, w_{1}, w_{2}\right)}{w_{2}^{\operatorname{ord}_{w_{2}}\left(\Omega_{2}\left(z, w_{1}, w_{2}\right)\right)}}\right)+N\left(r, \frac{1}{w_{2}^{\lambda_{22}-\operatorname{ord}_{w_{2}}\left(\Omega_{2}\right)}}\right) \\
\leq & \left(\lambda_{22}-\operatorname{ord}_{w_{2}}\left(\Omega_{2}\right)\right) N\left(r, w_{2}\right)+\lambda_{21} N\left(r, w_{1}\right) \\
& +T\left(r, \frac{1}{w_{2}^{\lambda_{22}-\operatorname{ord}_{w_{2}}\left(\Omega_{2}\right)}}\right)+S_{q}\left(r, w_{1}\right)+S_{q}\left(r, w_{2}\right) \\
\leq & \left(\lambda_{22}-\operatorname{ord}_{w_{2}}\left(\Omega_{2}\right)\right) N\left(r, w_{2}\right)+\lambda_{21} N\left(r, w_{1}\right) \\
& +\left(\lambda_{22}-\operatorname{ord}_{w_{2}}\left(\Omega_{2}\right)\right) T\left(r, w_{2}\right)+S_{q}\left(r, w_{2}\right)+S_{q}\left(r, w_{2}\right) .
\end{aligned}
$$

Combining (3.5) and (3.7), (3.6) and (3.8), respectively, we have

$$
\begin{aligned}
(1+ & \left.\lambda_{12}+\lambda_{11}-\operatorname{ord}_{w_{1}}\left(\Omega_{1}\right)\right) T\left(r, w_{1}\right) \\
< & \left(\lambda_{11}-\operatorname{ord}_{w_{1}}\left(\Omega_{1}\right)\right) N\left(r, w_{1}\right)+\lambda_{12} T\left(r, w_{2}\right) \\
& \quad+\left(\lambda_{11}-\operatorname{ord}_{w_{1}}\left(\Omega_{1}\right)\right) T\left(r, w_{1}\right)+S_{q}\left(r, w_{1}\right)+S_{q}\left(r, w_{2}\right)
\end{aligned}
$$

and

$$
\begin{aligned}
& \left(1+\lambda_{21}+\lambda_{22}-\operatorname{ord}_{w_{2}}\left(\Omega_{2}\right)\right) T\left(r, w_{2}\right) \\
& \quad<\left(\lambda_{22}-\operatorname{ord}_{w_{2}}\left(\Omega_{2}\right)\right) N\left(r, w_{2}\right)+\lambda_{21} T\left(r, w_{1}\right) \\
& \quad+\left(\lambda_{22}-\operatorname{ord}_{w_{2}}\left(\Omega_{2}\right)\right) T\left(r, w_{2}\right)+S_{q}\left(r, w_{1}\right)+S_{q}\left(r, w_{2}\right) .
\end{aligned}
$$

Suppose that $N\left(r, w_{1}\right)=S_{q}\left(r, w_{1}\right)$ and $N\left(r, w_{2}\right)=S_{q}\left(r, w_{2}\right)$, according to (3.9) and (3.10), we have

$$
\left(1+\lambda_{12}\right) T\left(r, w_{1}\right)<\lambda_{12} T\left(r, w_{2}\right)+S_{q}\left(r, w_{1}\right)+S_{q}\left(r, w_{2}\right)
$$

and

$$
\left(1+\lambda_{21}\right) T\left(r, w_{2}\right)<\lambda_{21} T\left(r, w_{1}\right)+S_{q}\left(r, w_{1}\right)+S_{q}\left(r, w_{2}\right) .
$$

That is,

$$
\left(1+\lambda_{12}+o(1)\right) T\left(r, w_{1}\right)<\left(\lambda_{12}+o(1)\right) T\left(r, w_{2}\right)
$$

and

$$
\left(1+\lambda_{21}+o(1)\right) T\left(r, w_{2}\right)<\left(\lambda_{12}+o(1)\right) T\left(r, w_{1}\right) .
$$

By (3.11) and (3.12), we conclude that

$$
1+\lambda_{12}+1+\lambda_{21}+o(1)<\lambda_{12}+\lambda_{21},
$$

which is impossible, we prove the assertion. 


\section{Proof of Theorem 2}

It follows by Lemma 1 that

$$
m\left(r, \frac{\Omega_{1}\left(z, w_{1}, w_{2}\right)}{w_{1}^{\lambda_{11}}}\right) \leq \lambda_{12} m\left(r, w_{2}\right)+S_{q}\left(r, w_{1}\right)
$$

and

$$
m\left(r, \frac{\Omega_{2}\left(z, w_{1}, w_{2}\right)}{w_{2}^{\lambda_{22}}}\right) \leq \lambda_{21} m\left(r, w_{1}\right)+S_{q}\left(r, w_{2}\right)
$$

for all $r$ outside of an exceptional set of finite logarithmic measure.

Suppose now that $\left(w_{1}(z), w_{2}(z)\right)$ is a finite-order meromorphic solution of (1.2). Denoting $C=\max _{j=1, \ldots, n}\left\{\left|c_{j}\right|\right\}$ in $\Omega_{1}\left(z, w_{1}, w_{2}\right)$ and $\Omega_{2}\left(z, w_{1}, w_{2}\right)$, by Lemma 2 , we obtain

$$
\begin{aligned}
N\left(r, \frac{\Omega_{1}\left(z, w_{1}, w_{2}\right)}{\left.w_{1}^{\lambda_{11}}\right) \leq}\right. & \lambda_{11}\left(N\left(|q| r, w_{1}\right)+N\left(r, \frac{1}{w_{1}}\right)\right) \\
& +\lambda_{12}\left(N\left(|q| r, w_{2}\right)+N\left(r, \frac{1}{w_{2}}\right)\right) \\
& +\lambda_{12} N\left(r, w_{2}\right)+S_{q}\left(r, w_{1}\right)+S_{q}\left(r, w_{2}\right) \\
= & \lambda_{11}\left(N\left(r, w_{1}\right)+N\left(r, \frac{1}{w_{1}}\right)\right)+\lambda_{12}\left(N\left(r, w_{2}\right)+N\left(r, \frac{1}{w_{2}}\right)\right) \\
& +\lambda_{12} N\left(r, w_{2}\right)+S_{q}\left(r, w_{1}\right)+S_{q}\left(r, w_{2}\right)
\end{aligned}
$$

for all $r$ outside of a set $E$ of finite logarithmic measure. By (4.1) and (4.3), we have

$$
\begin{aligned}
N\left(r, \frac{\Omega_{1}\left(z, w_{1}, w_{2}\right)}{w_{1}^{\lambda_{11}}}\right) \leq & \lambda_{11}\left(N\left(r, w_{1}\right)+N\left(r, \frac{1}{w_{1}}\right)\right) \\
& +\lambda_{12}\left(N\left(r, w_{2}\right)+N\left(r, \frac{1}{w_{2}}\right)\right)+S_{q}\left(r, w_{1}\right)+S_{q}\left(r, w_{2}\right) \\
\leq & \lambda_{12}\left(2 T\left(r, w_{1}\right)-m\left(r, w_{1}\right)\right)+\lambda_{12}\left(3 T\left(r, w_{2}\right)-2 m\left(r, w_{2}\right)\right) \\
& +S_{q}\left(r, w_{1}\right)+S_{q}\left(r, w_{2}\right)
\end{aligned}
$$

for all $r \notin E$. On the other hand, by (4.1) and (4.3),

$$
\begin{aligned}
& N\left(r, \frac{\Omega_{1}\left(z, w_{1}, w_{2}\right)}{w_{1}^{\lambda_{11}}}\right)+\lambda_{12} m\left(r, w_{2}\right) \\
& \quad \geq T\left(r, \frac{P_{1}\left(r, w_{1}\right)}{w_{1}^{\lambda_{11}} Q_{1} r, w_{1}}\right) \\
& \quad=\left(\max \left\{p_{1}, q_{1}+\lambda_{11}\right\}-\min \left\{\lambda_{11}, \operatorname{ord}_{w_{1}}\left(\Omega_{1}\right)\right\}\right) T\left(r, w_{1}\right)+S_{q}\left(r, w_{1}\right),
\end{aligned}
$$

where $r$ lies outside of a set $F$ of finite logarithmic measure. Combining inequalities (4.4) and (4.5) with the assumption in Theorem 2, we have

$$
\begin{gathered}
\left(\max \left\{p_{1}, q_{1}+\lambda_{11}\right\}-\min \left\{\lambda_{11}, \operatorname{ord}_{w_{1}}\left(\Omega_{1}\right)\right\}\right) T\left(r, w_{1}\right) \\
-\lambda_{12} m\left(r, w_{2}\right)+S_{q}\left(r, w_{1}\right)+S_{q}\left(r, w_{2}\right)
\end{gathered}
$$




$$
\begin{aligned}
\leq & \lambda_{11}\left(2 T\left(r, w_{1}\right)-m\left(r, w_{1}\right)\right)+\lambda_{12}\left(3 T\left(r, w_{2}\right)-2 m\left(r, w_{2}\right)\right) \\
& +S_{q}\left(r, w_{1}\right)+S_{q}\left(r, w_{2}\right) .
\end{aligned}
$$

Similarly, we obtain

$$
\begin{aligned}
(\max & \left.\left\{p_{2}, q_{2}+\lambda_{22}\right\}-\min \left\{\lambda_{22}, \operatorname{ord}_{w_{2}}\left(\Omega_{2}\right)\right\}\right) T\left(r, w_{2}\right) \\
& \quad-\lambda_{21} m\left(r, w_{1}\right)+S_{q}\left(r, w_{1}\right)+S_{q}\left(r, w_{2}\right) \\
\leq & \lambda_{22}\left(2 T\left(r, w_{2}\right)-m\left(r, w_{2}\right)\right)+\lambda_{21}\left(3 T\left(r, w_{1}\right)-2 m\left(r, w_{1}\right)\right) \\
& +S_{q}\left(r, w_{1}\right)+S_{q}\left(r, w_{2}\right) .
\end{aligned}
$$

By (4.6) and (4.7), we obtain

$$
\begin{aligned}
& \lambda_{11} m\left(r, w_{1}\right) \\
& \leq\left(2 \lambda_{11}-\left(\max \left\{p_{1}, q_{1}+\lambda_{11}\right\}-\min \left\{\lambda_{11}, \operatorname{ord}_{w_{1}}\left(\Omega_{1}\right)\right\}\right)+o(1)\right) T\left(r, w_{1}\right) \\
& \quad+\left(3 \lambda_{12}+o(1)\right) T\left(r, w_{2}\right)
\end{aligned}
$$

and

$$
\begin{aligned}
& \left(\left(\max \left\{p_{2}, q_{2}+\lambda_{22}\right\}-\min \left\{\lambda_{22}, \operatorname{ord}_{w_{2}}\left(\Omega_{2}\right)\right\}\right)-2 \lambda_{22}+o(1)\right) T\left(r, w_{2}\right) \\
& \quad \leq\left(3 \lambda_{21}+o(1)\right) T\left(r, w_{1}\right)-2 \lambda_{21} m\left(r, w_{2}\right)
\end{aligned}
$$

Combining (4.8) and (4.9), we have

$$
\begin{aligned}
\lambda_{11} m\left(r, w_{1}\right) & \\
\leq & \left(2 \lambda_{11}-\left(\max \left\{p_{1}, q_{1}+\lambda_{11}\right\}-\min \left\{\lambda_{11}, \operatorname{ord}_{w_{1}}\left(\Omega_{1}\right)\right\}\right)+o(1)\right) T\left(r, w_{1}\right) \\
& +\frac{3 \lambda_{12}\left(3 \lambda_{21}+o(1)\right) T\left(r, w_{1}\right)-6 \lambda_{12} \lambda_{21} m\left(r, w_{1}\right)}{\left(\max \left\{p_{2}, q_{2}+\lambda_{22}\right\}-\min \left\{\lambda_{22}, \operatorname{ord}_{w_{2}}\left(\Omega_{2}\right)\right\}\right)-2 \lambda_{22}},
\end{aligned}
$$

that is,

$$
\left(\lambda_{11}-\frac{6 \lambda_{12} \lambda_{21}}{B}\right) m\left(r, w_{1}\right) \leq\left(A-\frac{9 \lambda_{12} \lambda_{21}+o(1)}{B}\right) T\left(r, w_{1}\right),
$$

where $A=2 \lambda_{11}-\left(\max \left\{p_{1}, q_{1}+\lambda_{11}\right\}-\min \left\{\lambda_{11}, \operatorname{ord}_{w_{1}}\left(\Omega_{1}\right)\right\}\right)$ and $B=2 \lambda_{22}-\left(\max \left\{p_{2}, q_{2}+\lambda_{22}\right\}-\right.$ $\left.\min \left\{\lambda_{22}, \operatorname{ord}_{w_{2}}\left(\Omega_{2}\right)\right\}\right)$. Combining the assumption and (4.10), we have

$$
m\left(r, w_{1}\right)=S_{q}\left(r, w_{1}\right)
$$

for all $r$ outside of $E \cup F$, a set of finite logarithmic measure.

Similarly, we obtain

$$
m\left(r, w_{2}\right)=S_{q}\left(r, w_{2}\right)
$$

for all $r$ outside of $E \cup F$, we have proved the assertion. 


\section{Competing interests}

The authors declare that they have no competing interests.

\section{Authors' contributions}

All authors read and approved the final manuscript.

\section{Author details}

${ }^{1}$ Mathematics and Computer College, Northwest Minorities University, Lanzhou, Gansu 730030, China. ${ }^{2}$ Laiwu Vocational and Technical College, Laiwu, Shandong 271100, China. ${ }^{3}$ Shandong Transport Vocational College, Weifang, Shandong 261206, China.

\section{Acknowledgements}

Research was supported by the National Science Foundation of China (11161041), and the Fundamental Research Funds for the Central Universities (No. 31920130006) and Middle-Younger Scientific Research Fund (No. 12XB39)

Received: 25 March 2013 Accepted: 1 December 2013 Published: 06 Jan 2014

\section{References}

1. Hayman, W-K: Meromorphic Functions. Clarendon, Oxford (1964)

2. Laine, I: Nevanlinna Theory and Complex Differential Equations. de Gruyter, Berlin (1993)

3. Korhonen, R: A new Clunie type theorem for difference polynomials. J. Differ. Equ. Appl. 17(3), 387-400 (2011)

4. Zhang, JC, Wang, G, Chen, JJ, Zhao, RX: Some results on q-difference equations. Adv. Differ. Equ. 2012,191 (2012)

5. Barnett, D, Halburd, R-G, Korhonen, R-J, Morgan, W: Nevanlinna theory for the q-difference operator and meromorphic solutions of $q$-difference equations. Proc. R. Soc. Edinb. A 137(3), 457-474 (2007)

6. Hayman, W-K: On the characteristic of functions meromorphic in the plane and of their integrals. Proc. Lond. Math Soc. 14A, 93-128 (1965)

10.1186/1687-1847-2014-3

Cite this article as: Xu et al.: The solutions of one type $q$-difference functional system. Advances in Difference Equations $2014,2014: 3$

\section{Submit your manuscript to a SpringerOpen ${ }^{\ominus}$ journal and benefit from:}

- Convenient online submission

- Rigorous peer review

- Immediate publication on acceptance

- Open access: articles freely available online

- High visibility within the field

- Retaining the copyright to your article 